\title{
THE 3.3 MICRON PAH EMISSION OF THE MID-INFRARED EXCESS GALAXIES DISCOVERED BY THE AKARI MID-INFRARED ALL-SKY SURVEY
}

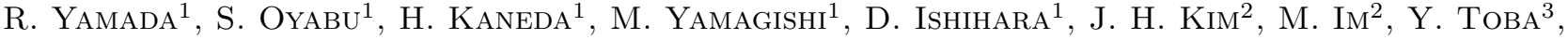 \\ AND H. MATSUHARA ${ }^{3}$ \\ ${ }^{1}$ Graduate school of science, Nagoya University, Nagoya, Aichi 464-8602, Japan \\ ${ }^{2}$ Department of Physics and Astrnomy, Seoul National University, Seoul 151-747, Korea \\ ${ }^{3}$ Institute of Space and Astronomical Science, Japan Aerospace Exploration Agency, 3-1-1 Yoshinodai, Chuo-ku, \\ 252-5210 Sagamihara, Japan \\ E-mail: ryamada@u.phys.nagoys-u.ac.jp \\ (Received July 01, 2012; Accepted August 12, 2012)
}

\begin{abstract}
We investigate the relation between star formation activity and PAH $3.3 \mu \mathrm{m}$ emission. Our targets are mid-infrared-excess galaxies selected from the AKARI all-sky survey point source catalog. We performed AKARI near-infrared spectroscopy for them. As a result, we obtained 2.5-5 $\mu \mathrm{m}$ spectra of 79 galaxies, and selected 35 star-forming galaxies out of them. Comparing the PAH 3.3 $\mu \mathrm{m}$ luminosities with the infrared luminosities, we find a linear correlation between them. However, by adding the results from literatures for luminous infrared galaxies and ultra-luminous infrared galaxies that are more luminous than our sample, the ratio of the PAH to the infrared luminosity is found to decrease towards the luminous end.
\end{abstract}

Key words: galaxies: active; galaxies: ISM; infrared: galaxies

\section{INTRODUCTION}

Polycyclic aromatic hydrocarbon (PAH) emission features are likely to be an indicator of star formation activities, because they are known to be ubiquitous in star-forming galaxies, and because they are destroyed by hard radiation from an AGN. In order to study availability of PAHs as a star-formation indicator, it is necessary to compare the PAH luminosity of the star-forming galaxies with the total infrared (IR) luminosity. Recent studies with Spitzer were performed in the mid-IR bands, but it is difficult to estimate the PAH luminosity because IRS slit spectroscopy misses a significant fraction of the radiation. Using AKARI near-IR spectroscopy with a 1 arcmin aperture, we can estimate the luminosity of a PAH feature at $3.3 \mu \mathrm{m}$, and compare it with the total IR luminosity. In addition, the PAH $3.3 \mu \mathrm{m}$ luminosity seems to reflect the radiation environment more sensitively than the other PAH emission features at large wavelengths due to very small PAHs which have a low heat capacity.

\section{OBSERVATION AND DATA ANALYSIS}

Our targets are selected from the AKARI mid-IR allsky point source catalog with the selection criterion $\mathrm{F}(9$ or $18 \mu \mathrm{m}) / \mathrm{F}(\mathrm{Ks})>2$ at galactic latitudes $|b| \geq 30^{\circ}$. Among them, we performed near-IR spectroscopy for 92 sources with AKARI during phase 3 mission program MSAGN (Oyabu et al., 2011). Out of them, 79 are galaxies and the others are stars.

We fitted a model to the $2.5-5 \mu \mathrm{m}$ spectra of the sample galaxies. For the model, we used a Drude profile for the PAH emission feature, Gaussian profiles for the $\mathrm{H}_{2} \mathrm{O}$ ice absorption feature $(\sim 3.05 \mu \mathrm{m})$ and $\mathrm{Br} \alpha$ emission line $(4.05 \mu \mathrm{m})$, and a power-law function for the continuum. As a result, we detect the PAH $3.3 \mu \mathrm{m}$ emission feature $(>3 \sigma)$ from 47 galaxies out of 79 .

We excluded sources likely contaminated by AGN 

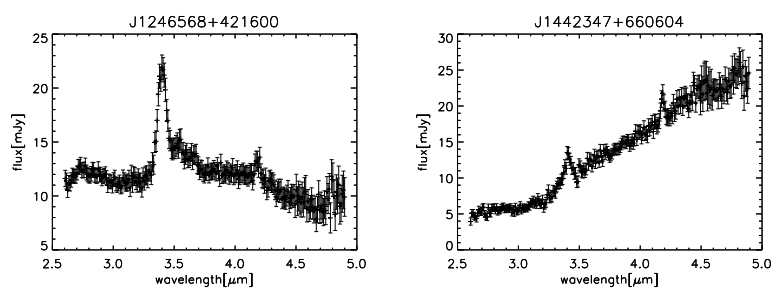

Fig. 1. Examples of spectra for star-forming galaxies (left) and AGN dominated galaxies (right).

activity based on the equivalent width of the PAH emission feature $(<40 \mathrm{~nm})$ (Moorwood, 1986; Imanishi et al., 2008) and the power-law index of the continuum (>1) (Imanishi et al., 2010). Of the 47 galaxies, 35 are considered to be star-formation dominated galaxies (the left panel of Fig. 1), and the others show the signature of AGN activity (the right of Fig. 1).

\section{DISCUSSION AND CONCLUSION}

As for the 35 star-forming galaxies, comparing the PAH luminosity, $L_{\mathrm{PAH} 3.3}$, with the total IR luminosity, $L_{\mathrm{IR}}$, estimated from IRAS fluxes and the broadband flux equation of Table 1 in Sanders \& Mirabel (1996), we find a good correlation between them. It is quite reasonable because both are tracers of starformation activity. The averaged value of $L_{\mathrm{PAH} 3.3} / L_{\mathrm{IR}}$ is $\sim 10^{-3}$ in the $L_{\mathrm{IR}}$ range of $10^{8}-10^{11.5} L_{\odot}$, which is a typical value for starburst galaxies. However, in Fig. 2, combining our result with the data of luminous infrared galaxies (LIRGs) and ultra-luminous infrared galaxies (ULIRGs) from Imanishi et al. (2008, 2010), we find that $L_{\mathrm{PAH} 3.3} / L_{\mathrm{IR}}$ decreases with $L_{\mathrm{IR}}$ at $\gtrsim 10^{11} L_{\odot}$. Imanishi et al. (2010) reported the decreases in ULIRGs and concluded that the AGN contributions were a major cause. However, we see this decline in star-forming ULIRGs. Possible causes of this decline are (1) dust extinction, (2) destruction of PAHs by intense UV radiation, and (3) the scarcity of PAHs.

If this decline results from the dust extinction, the $\operatorname{Br} \alpha$ emission line should be absorbed as well as the $\mathrm{PAH}$ emission. However, we find that there is no decreasing trend in the $L_{B r \alpha} / L_{\mathrm{IR}}-L_{\mathrm{IR}}$ plot, such as seen in Fig. 2, which indicates that the extinction is not a major cause.

Next, we compare $L_{\mathrm{PAH} 3.3} / L_{\mathrm{IR}}$ with the dust temperature and the dust mass, which are obtained by the ratio of IRAS 60 to $100 \mu \mathrm{m}$ fluxes with the emissivity index $\beta=1$. There is no clear correlation be-

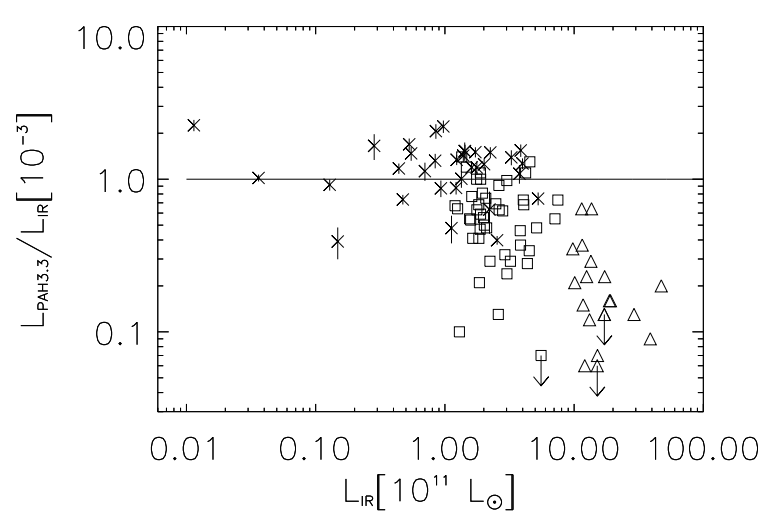

Fig. 2. $L_{\mathrm{PAH} 3.3} / L_{\mathrm{IR}}$ plotted against $L_{\mathrm{IR}}$ for the galaxies with no signature of AGN activity. The crosses correspond to our sample, while the squares and triangles are the LIRGs and ULIRGs from Imanishi et al. (2008, 2010), respectively. The solid line shows a typical ratio of starburst galaxies (Mouri et al., 1990).

tween $L_{\mathrm{PAH} 3.3} / L_{\mathrm{IR}}$ and the dust temperature, which means that the UV radiation field does not affect the $\mathrm{PAH}$ abundance. On the other hand, there is a tight correlation between $L_{\mathrm{PAH} 3.3} / L_{\mathrm{IR}}$ and the dust mass. Thus ULIRGs contain high dust masses. We therefore conclude that the scarcity of PAHs in ULIRGs relative to large grains is a major cause of the decline in $L_{\mathrm{PAH} 3.3} / L_{\mathrm{IR}}$.

\section{REFERENCES}

Imanishi, M., et al., 2008, Systematic Infrared 2.5$5 \mu \mathrm{m}$ Spectroscopy of Nearby Ultraluminous Infrared Galaxies with AKARI, PASJ, 60, S489

Imanishi, M., et al., 2010, AKARI IRC Infrared 2.5-5 $\mu \mathrm{m}$ Spectroscopy of a Large Sample of Luminous Infrared Galaxies, ApJ, 721, 1233

Moorwood, A. F. M., 1986, 3.28 Micron Feature and Continuum Emission in Galaxy Nuclei, A\&A, 166,4

Mouri, H., et al., 1990, A Relation between H2 V = 1-0 S(1) and 3.28 Micron Emission in Seyfert and Starburst Galaxies, ApJ, 356, L39

Oyabu, S., et al. 2011, AKARI Detections of Hot Dust in Luminous Infrared Galaxies. Search for Dusty Active Galactic Nuclei, A\&A 529, 122

Sanders, D. B. \& Mirabel, I. F., 1996, Luminous Infrared Galaxies, ARA\&A, 34, 749 